\title{
Multiple joint pain following steroid therapy
}

\author{
D McClelland, R L Morgan-Jones, C Wynn-Jones
}

A 28-year-old man presented to the orthopaedic out-patient department complaining of increasing pain in his left knee. Physical examination confirmed medial knee joint tenderness. He had recently completed an 8-month course of steroids for an episode of focal glomerulosclerosis which had caused generalised oedema. There was no other relevant history. Plain X-rays were taken (figure 1). Initial orthopaedic management was symptomatic pain relief only. The patient returned 9 months later complaining of pain in his right knee and both elbows. Over the following 5 months he also developed pain in both shoulders and hips (figure 2).

The pain from both hips increased and 12 months after first presentation he underwent bilateral pertrochanteric proximal femoral osteotomies in an attempt to produce a more congruent, less symptomatic joint. In view of his young age, joint replacement was thought inappropriate. Postoperatively his mobility improved and his hip pain settled.

Five years after initial presentation, the pain in his right hip returned and at this stage he underwent an uncemented total hip replacement with good results. Two years later he underwent the same procedure on the left side, again for increasing pain. Currently his elbows and shoulders are quiescent, but both knees have become increasingly painful. The patient will continue under close follow-up and appreciates that he probably faces multiple future procedures.

\author{
Hartshill Orthopaedic \\ Hospital, \\ Stoke-on-Trent, \\ Staffordshire ST4 7NZ, \\ UK \\ D McClelland \\ R L Morgan-Jones \\ C Wynn-Jones \\ Correspondence to \\ Mr RL Morgan-Jones, \\ Laurel House, Ellesmere, \\ Shropshire SY12 9AP, UK
}

Accepted 23 April 1998

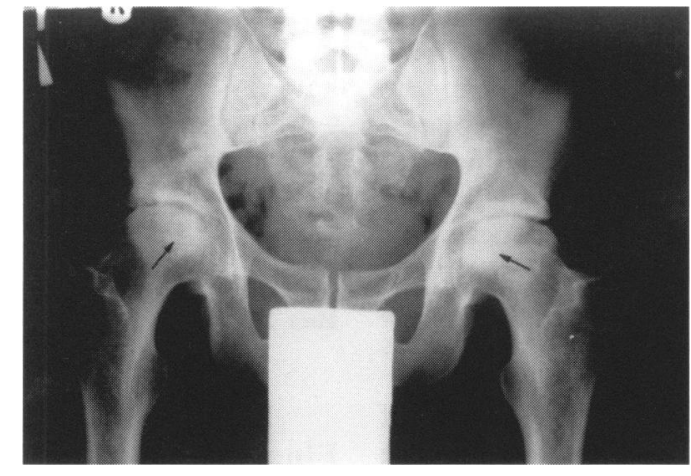

Figure 1

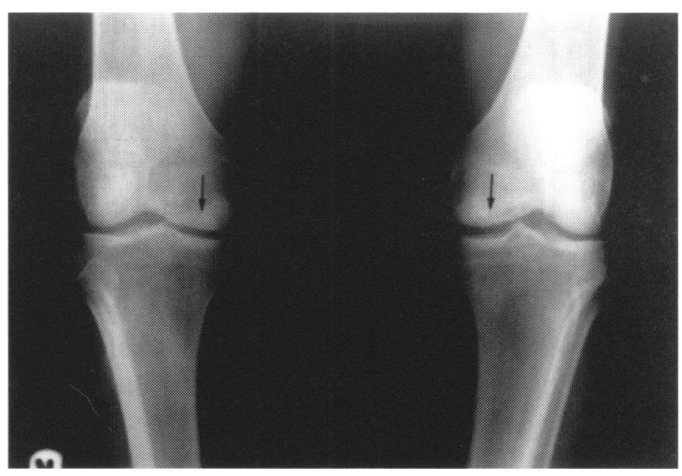

Figure 2

\section{Questions}

1 Describe the relevant features on the X-rays.

2 What are the recognised causes of this condition? 
Answers

QUESTION 1

The X-rays show loss of joint space, sclerosis of subchondral bone, osteophytes, and cysts.

QUESTION 2

The recognised causes of this condition are trauma (disruption of blood supply), steroid therapy, alcoholism, Gaucher's disease, sickle cell disease, Caisson's disease, atheromatous / embolic arterial disease, and post-irradiation vascular thrombosis.

\section{Discussion}

Osteonecrosis (avascular necrosis) results from the loss of blood supply to bone causing local bone death. When this occurs in bone adjacent to a joint there is loss of support and collapse of the articular cartilage resulting in secondary osteoarthritis. Ficat ${ }^{1}$ classified osteonecrosis of the hip into stages, based on clinical findings and radiographic appearances (table). Aseptic osteonecrosis was first described in 1888 by Franz Konig. ${ }^{2}$ Since then multiple causes have been identified, ${ }^{34}$ including steroids, alcohol abuse, Caisson's disease and renal failure. Steroid-induced osteonecrosis has been recognised for over 40 years and cases after both short- and long-term usage are documented. ${ }^{45}$ Cases after short-term usage tend to follow high-dose steroid treatment. ${ }^{6}$ However, the correlation between steroid dose and appearance or degree of osteonecrosis is unclear. ${ }^{5}$ Steroid-induced osteonecrosis is usually isolated to a single joint but, as in this case, can rarely become disseminated. The joints commonly affected are the femoral head, humeral head, knee, talus and capitellum with lesions localised to the subchondral region. ${ }^{4}$

Three theories exist as to the pathogenesis of osteonecrosis (box 1). The theory involving altered fat metabolism has most scientific support. Wang et $a l,{ }^{6}$ showed that steroid administration resulted in a state of hypercholesterolaemia and an increased incidence of fat
Table Ficat's stages of hip avascular necrosis

\begin{tabular}{lll}
\hline Stage & Pain & $X$-Ray \\
\hline I & Minimal & Normal \\
II & Moderate & Porosis/sclerosis \\
III & Advanced & Flat/crescent sign \\
IV & Severe & Acetabular changes \\
\hline
\end{tabular}

embolisation resulting in occlusion of subchondral arterioles with subsequent ischaemic necrosis. An increase in marrow fat cell size also occurs leading to an increase in the intraosseous pressure. Vascular compression results, which in turn causes marrow and bone death. ${ }^{6}$

\section{Pathogenesis of steroid-induced osteonecrosis}

- steroid-induced hypercoagulable state resulting in 'sludging' within vessels

- steroid-induced osteoporosis

- steroid-induced fat cell changes with resulting fat micro-emboli and increased marrow fat cell size

Box 1

\section{Learning points}

- consider avascular necrosis when persistent joint pains occur in patients on steroid therapy

- early diagnosis improves the chance of successful intervention

Box 2

\section{Final diagnosis}

Multifocal avascular necrosis.

Keywords: steroid therapy; avascular necrosis
1 Ficat RP, Arlet J. Ischaemia and necroses of bone. Baltimore: Williams \& Wilkins, 1980.

2 McArthy EF. Aseptic necrosis of bone. Clin Orthopaed Rel Res 1992;168:216-21.

3 Bernard J. Epidemiology of traumatic and non traumatic osteonecrosis. Clin Orthopaed Rel Res 1978;130:51-67.

4 Ibels LS, Alfrey AC, Huffer WF. Aseptic necrosis of bone following renal transplantation: experience in 194 transplant recipients and review of the literature. Medicine 1978;57:2545.
5 O'Brien TJ, Mack GR. Multi focal osteonecrosis after short term high dose corticosteroid therapy. Clin Orthopaed Rel Res 1992;279:176-9.

6 Wang G-J, Sweet DE, Reger SI, Thompson RC. Fat cell changes as a mechanism of avascular necrosis of the femoral head in cortisone-treated rabbits. $\mathcal{F}$ Bone foint Surg 1977;59-A:729-35. 